\title{
The level of distribution of the special values of $L$-functions
}

\author{
by
}

\author{
Ritabrata Munshi (Piscataway, NJ)
}

1. Introduction. Let $E$ be an elliptic curve over $\mathbb{Q}$ of conductor $N$, root number $\omega$, and defined by the Weierstrass equation $y^{2}=f(x)$. For any square-free $d$ relatively prime to $N$, the root number of the twisted curve $E^{d}: d y^{2}=f(x)$ is given by $\omega_{d}=\omega \chi_{d}(-N)$, where $\chi_{d}$ denotes the quadratic character associated with the field $\mathbb{Q}(\sqrt{d})$. Then depending on $N$ and $\omega$ one may appropriately choose a congruence class modulo $4 N$ such that for each $d$ in the congruence class the root number $\omega_{d}$ is either always 1, or always -1 . In the light of the deep results of Kolyvagin, Gross and Zagier [3], it is natural to study the central values $\left\{L\left(1, E^{d}\right)\right\}$ for the family of twists with fixed root number 1 , as in this case the nonvanishing of $L\left(1, E^{d}\right)$ implies that there are at most finitely many $\mathbb{Q}$-rational points on the elliptic curve $E^{d}$. On the other hand, when the root number is -1 , then the central values vanish and one studies the sequence of derivatives $\left\{L^{\prime}\left(1, E^{d}\right)\right\}$ with the hope of proving that some or many of them are nonvanishing. Of course, then it follows that the corresponding elliptic curve has Mordell-Weil rank one, and consequently infinitely many rational points. Such nonvanishing results are well known when the only restriction on $d$ is a congruence condition. This was first established by Murty and Murty [11], and almost simultaneously, but independently and via a totally different method, by Bump, Friedberg and Hoffstein [1]. Later Iwaniec [7] gave a quantitative version of this result.

Goldfeld has proposed a strong conjecture regarding the distribution of $r_{d}=\operatorname{ord}_{s=1} L\left(s, E^{d}\right)$ in the family of all quadratic twists $\left\{E^{d}\right\}$ (with $d$ square-free and $(d, N)=1)$. Let $n(X)$ denote the number of curves in the above family with $d<X$. Then the conjecture predicts that

$$
\sum_{\substack{d \leq X \\ r_{d}=i}} 1 \sim \frac{1}{2} n(X)
$$

2000 Mathematics Subject Classification: 11G40, 11N36, 11F67.

Key words and phrases: elliptic curve, $L$-function, sieves. 
for $i=0,1$. This is an important unsolved problem and any partial result towards this conjecture will be highly welcome. One consequence of the conjecture is that a positive proportion of the central values $\left\{L\left(1, E^{d}\right)\right\}$ (respectively central derivatives $\left\{L^{\prime}\left(1, E^{d}\right)\right\}$ ) do not vanish. The first example illustrating this phenomenon for $L\left(1, E^{d}\right)$ was given by James [9]. In his example the elliptic curve $E$ is the -1 twist of the modular curve $X_{0}(14)$. Vatsal [16] has established that the elliptic curve $E=X_{0}(19)$ has a positive proportion of nonvanishing central derivatives $L^{\prime}\left(1, E^{d}\right) \neq 0$. For general elliptic curves Ono [12] has established that the number of $d<X$ with $L\left(1, E^{d}\right) \neq 0$ is at least $X /(\log X)^{\alpha}$ for some $\alpha<1$. In the case of central derivatives the best known result is due to Perelli and Pomykała [14] who show that there are at least $X^{1-\varepsilon}$ such twists for any $\varepsilon>0$. One should note that Ono in [12] employs the theorem of Waldspurger relating the central value $L\left(1, E^{d}\right)$ with the $d$ th Fourier coefficient of a half-integral weight modular form, and this is why he gets a stronger result in the case of central values. The method used in [14], on the other hand, is totally analytic-number-theoretic and is based on the large sieve inequalities.

Our interest in the problem of nonvanishing of special values stems from two different arithmetic applications. First we are motivated by the following question: "Given an elliptic curve $E$ are there infinitely many primes $p$ such that the quadratic twists $E^{p}$ have rank zero?" In the same spirit one may ask for prime twists of rank one. Ono and Skinner [13] settled the above question affirmatively by transferring the problem to that about Fourier coefficients of a half-integral weight modular form using the theorem of Waldspurger. In this way they bypassed all the relevant problems that one faces while dealing with such sieve-theoretic questions. However, since there is no satisfactory analogue of Waldspurger's result in the case of derivatives, it is still important to study the above question using sieve theory, that is to say, to study the level of distribution of the special values of $L$-functions. Our second motivation comes from a possible Diophantine application. Consider the family of elliptic curves $\left\{E_{t}\right\}_{t \in \mathbb{Q}}$ where

$$
E_{t}: \quad\left(t^{2}+1\right) y^{2}=f(x),
$$

and $f$ is a cubic polynomial. To show that the above algebraic family has infinitely many fibers of rank 1 , it is enough to show that there are infinitely many square-free integers $n$ such that all the prime factors of $n$ are of the form $4 k+1$, and the elliptic curve $E^{n}: n y^{2}=f(x)$ has rank 1. Such problems fall naturally in the realm of sieve theory.

In this paper we study the distribution of the finite sequence $\left\{s_{d}\right\}$, where

$$
s_{d}=\mu(d)^{2} \psi_{A}(d)\left(1-\omega_{d}\right) L^{\prime}\left(1, E^{d}\right) F(d / Y) .
$$

Here $F$ is a nonnegative-valued compactly supported smooth function, $A$ is 
a square-free odd integer coprime to $N$, and

$$
\psi_{A}(d)=\prod_{p \mid A} \frac{1}{2}\left\{1+\left(\frac{d}{p}\right)\right\}= \begin{cases}1 & \text { if }\left(\frac{d}{p}\right)=1 \text { for all } p \mid A, \\ 0 & \text { otherwise. }\end{cases}
$$

So $\psi_{A}$ is the indicator function for the set of integers which are quadratic residues modulo each prime divisor of $A$. This is an innocent sieve and it naturally appears in many contexts, e.g. while considering numbers represented by the form $u^{2}+A v^{2}$, so we incorporate it in the definition of the sequence. Then for any square-free $\xi$ coprime to $2 N A$ we show that

$$
\sum_{d \equiv 0(\bmod \xi)} s_{d}=g(\xi)\left(c_{1} \log Y+c_{2}\right) Y+h(\xi) c_{3} Y+r_{\xi},
$$

where $c_{1}, c_{2}$ and $c_{3}$ are constants which depend only on the elliptic curve, the smooth cut-off function $F$ and the number $A$. Moreover, $c_{1}$ is nonzero, and $g(\xi)$ is a nice multiplicative function, $h(\xi)$ is almost multiplicative and the error term $r_{\xi}$ satisfies

$$
\sum_{\xi<Y^{\theta}}\left|r_{\xi}\right| \ll Y^{3 / 4+\theta / 2+\varepsilon}
$$

The nonnegativity of the terms in the sequence $\left\{s_{d}\right\}$ follows from the result of Gross and Zagier, and so the sequence is nicely adapted to sieve methods. One may object that the density function is not completely multiplicative, but we will see that the almost multiplicativity of $h(\xi)$ suffices for our purpose. In this context the above result then says that the level of distribution of the sequence of central derivatives is almost $\sqrt{Y}$ (or "one-half").

Similarly, one may study the sequence of central values $\left\{\widetilde{s}_{d}\right\}$, where

$$
\widetilde{s}_{d}=\mu(d)^{2} \psi_{A}(d)\left(1+\omega_{d}\right) L\left(1, E^{d}\right) F(d / Y) .
$$

In this case the asymptotic behaviour is given by

$$
\sum_{d \equiv 0(\bmod \xi)} \widetilde{s}_{d}=\widetilde{g}(\xi) c_{4} Y+\widetilde{r}_{\xi},
$$

where $\widetilde{g}(\xi)$ is a nice multiplicative function, $c_{4}$ is a nonzero constant and the error term $\widetilde{r}_{\xi}$ satisfies

$$
\sum_{\xi<Y^{\theta}}\left|\widetilde{r}_{\xi}\right| \ll Y^{3 / 4+\theta / 2+\varepsilon} .
$$

So this sequence is more natural for sieve techniques. However, we choose to work with the sequence of central derivatives because of two reasons: (1) to investigate any new subtlety that the term $h(\xi)$ may present, and (2) the derivatives are more interesting from the point of view of Diophantine geometry. 
2. Statement of results. We start with the approximate functional equation which gives a representation of the $L$-values at the central point in terms of certain rapidly converging series. In the case of the derivative, when the root number is -1 we have

$$
L^{\prime}\left(1, E \chi_{d}\right)=2 \mathcal{A}\left(d \sqrt{N}, \chi_{d}\right),
$$

where

$$
\mathcal{A}(X, \chi)=\sum_{n=1}^{\infty} \frac{a_{n} \chi(n)}{n} V\left(\frac{2 \pi n}{X}\right) .
$$

Here $a_{n}$ 's are the Fourier coefficients of the modular form associated to $E$ (by [17]), and $V$ is the incomplete gamma function defined by

$$
V(X)=\int_{X}^{\infty} e^{-t} \frac{d t}{t}=\frac{1}{2 \pi i} \int_{\left(\frac{3}{4}\right)} \frac{\Gamma(s)}{s} X^{-s} d s .
$$

It is also advisable for the sake of notational simplicity to put certain assumptions on the conductor $N$ and the root number $\omega$ of the elliptic curve $E$. We assume that $N \equiv 7(\bmod 8)$ and that $\omega=1$. Let $A$ be a square-free odd integer coprime to $N$. Then we run $d$ over the set of square-free odd integers coprime to $2 N A$, and such that $\left(\frac{d}{N}\right)=1$. Hence the root number of the twisted curve $E^{d}$ is -1 . Let $F$ be a compactly supported nonnegative-valued smooth function. Then we define a finite sequence of nonnegative quantities by

$$
s_{d}=\mu(d)^{2} \psi_{A}(d)\left(1+\left(\frac{d}{N}\right)\right) L^{\prime}\left(1, E^{d}\right) F(d / Y)
$$

when $(d, 2 N A)=1$, and 0 otherwise. Now we state our main result.

TheOREM 1. Suppose $\xi$ is a square-free odd integer coprime to NA. Then we have

$$
\sum_{d \equiv 0(\bmod \xi)} s_{d}=g(\xi)\left(c_{1} \log Y+c_{2}\right) Y+h(\xi) c_{3} Y+r_{\xi},
$$

where $c_{1}, c_{2}$ and $c_{3}$ are constants which depend only on the elliptic curve, the smooth cut-off function $F$ and the number $A$. Moreover, $c_{1}$ is nonzero, and the error term $r_{\xi}$ satisfies

$$
\sum_{\xi<Y^{\theta}}\left|r_{\xi}\right| \ll_{\varepsilon} Y^{3 / 4+\theta / 2+\varepsilon} .
$$

The multiplicative function $g(\xi)=\prod_{p \mid \xi} g(p)$ is defined by

$$
g(p)=\frac{1}{p}\left(1+\frac{1}{p}+\frac{a_{p^{2}}}{p^{2}}+\frac{a_{p^{4}}}{p^{4}}+\cdots\right)^{-1} .
$$


The function $h(\xi)$ is almost multiplicative and is given by

$$
g(\xi) \sum_{p \mid \xi}\left(1+\frac{1}{p}+\frac{a_{p^{2}}}{p^{2}}+\frac{a_{p^{4}}}{p^{4}}+\cdots\right)^{-1}\left(\frac{2 a_{p^{2}}}{p^{2}}+\frac{4 a_{p^{4}}}{p^{4}}+\cdots\right) \log p .
$$

In [6] Hoffstein and Luo have stated a similar result for the sequence of central values. Their proof is based on the theory of metaplectic forms and is much more abstruse compared to our direct analytic-number-theoretic approach. Their argument, in principle, should work for derivatives too, but that would require a careful study of the related metaplectic Eisenstein series. Unfortunately, the full details of their proof never appeared. The following two corollaries are immediate from the above theorem and the standard results on combinatorial sieves $([2],[4],[8])$. We just make the following comment. The term $h(\xi)$, though not multiplicative, is of the form $h(\xi)=g(\xi) \sum_{p \mid \xi} w(p)$, where $g(\xi)$ is multiplicative and $w(p)=O\left(\frac{\log p}{p}\right)$. This follows from the standard bound for the Fourier coefficients. Such almost-multiplicative functions can be dealt with by using sieves. Now for any set $\mathcal{P}$ of prime numbers, set

$$
P(z)=\prod_{\substack{p<z \\ p \in \mathcal{P}}} p
$$

COROLlary 2. Let $\mathcal{P}$ be a set of primes obtained by deleting a finite number of primes. Suppose that $\mathcal{P}$ does not contain any of the prime factors of $2 N A$. Then

$$
\sum_{(d, P(z))=1} s_{d} \gg Y
$$

if $z=Y^{\theta}$ with $\theta<1 / 4$.

From the above corollary we get results of the following form: there exist infinitely many $d$ with at most four prime factors, such that the Mordell-Weil rank of the twisted elliptic curve $E^{d}$ is one. It should be possible to reduce the number of prime divisors to three via weighted sieves. Of course, cutting short the number to one will demand overcoming formidable difficulties, given that the level of distribution that we achieve is only at most one-half.

COROllary 3. Let $\mathcal{P}$ be the set of primes $p$ such that $\chi_{A}(p)=-1$. Then

$$
\sum_{(d, P(z))=1} s_{d} \gg Y \sqrt{\log Y}
$$

if $z=Y^{\theta}$ with $\theta<1 / 2$.

The condition $\psi_{A}(d)=1$ implies that $d$ has an even number of prime factors from the set $\mathcal{P}$. Hence to make $d$ free of primes from $\mathcal{P}$ we need to take the sieving range $z=\sqrt{Y}$, which is just beyond the range allowed by the level 
of distribution proved in the theorem. So it will be very interesting if one can improve the level of distribution by even "an epsilon". Let us also remark that using the above corollary we get rank one twists by discriminants having either none or exactly two prime factors $p_{1}, p_{2}$ with $\chi_{A}\left(p_{1}\right)=\chi_{A}\left(p_{2}\right)=-1$. These two primes are very near to each other and one hopes that they contribute a small portion compared to the contribution of terms having no such primes. However, we have not been able to prove this. Now we move to the next important consequence which improves on the quantitative result given by Iwaniec in [7].

Corollary 4. Let $E$ be an elliptic curve with root number 1 . Then for some constants $a \neq 0$ and $b$ we have

$$
\sum_{(d, 2 N)=1} \mu(d)^{2}\left(1-\omega_{d}\right) L^{\prime}\left(1, E^{d}\right) F(d / Y)=a Y \log Y+b Y+O\left(Y^{3 / 4+\varepsilon}\right) .
$$

Finally, a few words about the proof of the theorem. The proof closely follows the treatment given by Soundararajan in [15]. The large sieve inequality proved by Heath-Brown in [5] is one of the main ingredients in [15]. Perelli and Pomykała [14] have adapted Heath-Brown's work in the elliptic curve scenario. In particular, using large sieve they prove the following bounds:

$$
\sum_{d \leq D}^{b}\left|L^{\prime}\left(1, E \chi_{d}\right)\right|^{2} \ll_{\varepsilon} D^{1+\varepsilon}
$$

and

$$
\sum_{d \leq D}^{b}\left|L\left(\sigma+i t, E \chi_{d}\right)\right|^{2} \ll_{\varepsilon}\left(D+(D \tau)^{3-2 \sigma}\right)(D \tau)^{\varepsilon}
$$

(where $\tau=|t|+1$ ) uniformly for $1 \leq \sigma \leq 3 / 2$ and $t \in \mathbb{R}$. Here $\sum^{b}$ indicates that the sum is over square-free integers. These two bounds will be crucial in our proof.

It is desirable to improve these bounds so that the factor $D^{\varepsilon}$ is replaced by a power of $\log D$. Such an improvement will increase the level of distribution albeit by an "epsilon", but that may turn out to be enough to get the Diophantine application that we described in the introduction.

3. A mean value estimate-I. We start by repeating the various assumptions that we have made. $E$ is an elliptic curve with conductor $N$ which is congruent to 7 modulo 8 . Also, we are assuming that the root number of the elliptic curve is 1 . We twist $E$ by $2 d$, where $d$ is an odd positive integer coprime to $N A$ and $A$ is a fixed odd integer coprime to $N$. Then the root number of $E^{2 d}$ is -1 and we have the expression

$$
L^{\prime}\left(1, E \chi_{8 d}\right)=2 \mathcal{A}\left(8 d \sqrt{N}, \chi_{8 d}\right) .
$$


Since the quadratic field $\mathbb{Q}(\sqrt{2 d})$ has discriminant $8 d$ we are writing $\chi_{8 d}$ for the associated quadratic character. Now we fix a square-free odd positive integer $\xi$ co-prime to $N A$, and a divisor $\alpha$ of $A$. Our goal in this section is to estimate the following:

$$
S(\alpha):=\sum_{(d, 2 N A \xi)=1} \mu(d)^{2}\left(\frac{d}{\alpha}\right) \mathcal{A}\left(8 d \xi \sqrt{N}, \chi_{8 d \xi}\right) F\left(\frac{d \xi}{Y}\right),
$$

where $F$ is a nonnegative-valued smooth function supported on the interval $(1,2)$. The importance of these parameters will become clear later.

As $\mu(d)^{2}=\sum_{l^{2} \mid d} \mu(l)$, by expanding $\mathcal{A}$ and interchanging the order of summation, we get

$$
S(\alpha)=\sum_{n} \frac{a_{n}}{n} \sum_{\substack{l \leq X \\(l, 2 N A \xi n)=1}} \mu(l)\left(\frac{2 \xi}{n}\right) \sum_{(d, 2 N A \xi)=1}\left(\frac{d}{\alpha n}\right) F_{n}\left(\frac{d \xi l^{2}}{Y}\right)+R(\alpha),
$$

where

$$
\begin{aligned}
& F_{n}(t)=F(t) V\left(\frac{2 \pi n}{8 t Y \sqrt{N}}\right), \\
& R(\alpha)=\sum_{(d, 2 N A \xi)=1}\left(\sum_{\substack{l^{2} \mid d \\
l>X}} \mu(l)\right)\left(\frac{d}{\alpha}\right) \mathcal{A}\left(8 d \xi \sqrt{N}, \chi_{8 d \xi}\right) F\left(\frac{d \xi}{Y}\right) .
\end{aligned}
$$

The parameter $X$ will be chosen later. It is easy to see that $R(\alpha)$ is much smaller than the expected leading term and so we leave it as it is for the time being, and continue our analysis with the first term. Treating the coprimality condition in the inner sum using the Möbius function we write

where

$$
S(\alpha)=\sum_{\delta \mid N A \xi} \mu(\delta)\left(\frac{\delta}{\alpha}\right) S(\alpha, \delta)+R(\alpha),
$$

$$
S(\alpha, \delta)=\sum_{n} \frac{a_{n}}{n} \sum_{\substack{l \leq X \\(l, 2 N A \xi n)=1}} \mu(l)\left(\frac{2 \xi \delta}{n}\right) \sum_{d \text { odd }}\left(\frac{d}{\alpha n}\right) F_{n}\left(\frac{l^{2} \xi \delta d}{Y}\right) .
$$

We will often write $\delta=\delta_{1} \delta_{2}$, where $\delta_{1} \mid N A$ and $\delta_{2} \mid \xi$. Also, it is clear that we are just required to consider $\delta$ coprime to $\alpha$. Now, we apply the Poisson summation formula to $S(\alpha, \delta)$, and obtain

$$
\begin{aligned}
S(\alpha, \delta)= & \frac{Y}{\xi \delta \alpha} \sum_{n} \frac{a_{n}}{n^{2}} \sum_{\substack{l \leq X \\
(l, 2 N A \xi n)=1}} \frac{\mu(l)}{2 l^{2}}\left(\frac{2 \xi \delta}{n}\right)\left(\frac{2}{\alpha n}\right) \\
& \times \sum_{k}(-1)^{k} G_{k}(\alpha n) \widetilde{F}_{n}\left(\frac{k Y}{2 l^{2} \xi \delta \alpha n}\right),
\end{aligned}
$$


which we write as $P(\alpha, \delta)+R(\alpha, \delta)$, where $P(\alpha, \delta)$ is the principal term arising from the $k=0$ term, and $R(\alpha, \delta)$ includes all the nonzero $k$-terms. Again, $R(\alpha, \delta)$ will contribute only to the error term, and will be evaluated in a later section. Here $G_{k}(n)$ is a Gauss-type sum given by

$$
G_{k}(n)=\varepsilon_{n}^{-1} \sum_{a \bmod n}\left(\frac{a}{n}\right) e\left(\frac{a k}{n}\right)
$$

where

$$
\varepsilon_{n}^{-1}=\frac{1-i}{2}+\left(\frac{-1}{n}\right) \frac{1+i}{2}
$$

and the Fourier transform is defined in the following way:

$$
\widetilde{F}(y)=\int_{-\infty}^{\infty}(\cos (2 \pi x y)+\sin (2 \pi x y)) F(x) d x .
$$

4. The main term. Note that $G_{0}(\alpha n)=\phi(\alpha n)$ if $\alpha n$ is a square and $G_{0}(\alpha n)=0$ otherwise. So it follows that

$$
P(\alpha, \delta)=\frac{Y}{\xi \delta \alpha} \sum_{n \alpha=\square} \frac{a_{n} \phi(n \alpha)}{n^{2}} \sum_{\substack{l \leq X \\(l, 2 N A \xi n)=1}} \frac{\mu(l)}{2 l^{2}}\left(\frac{2 \xi \delta}{n}\right)\left(\frac{2}{\alpha n}\right) \widetilde{F}_{n}(0) .
$$

( $k=\square$ means that $k$ is a square of an integer.)

Then completing the $l$-sum, and using the well known bound for $a_{n}$, we deduce that

$$
\begin{aligned}
P(\alpha, \delta)= & \frac{Y}{2 \xi \delta \alpha} \sum_{n \alpha=\square} \frac{a_{n} \phi(n \alpha)}{n^{2}}\left(\frac{2 \xi \delta}{n}\right)\left(\frac{2}{\alpha n}\right) \prod_{(p, 2 N A \xi n)=1}\left(1-\frac{1}{p^{2}}\right) \widetilde{F}_{n}(0) \\
& +O_{\varepsilon}\left(\frac{Y^{1+\varepsilon}}{X \xi \delta}\right) .
\end{aligned}
$$

Now, $\alpha n=\square$ iff $n=\alpha m^{2}$ (as $\alpha$ is square-free), and in that case

$$
\left(\frac{2 \xi \delta}{n}\right)\left(\frac{2}{\alpha n}\right)= \begin{cases}\left(\frac{2 \xi \delta}{\alpha}\right) & \text { if }(2 \xi \delta, m)=1 \\ 0 & \text { otherwise. }\end{cases}
$$

Also,

$$
\phi\left(\alpha^{2} m^{2}\right) \prod_{\substack{p \mid m \\(p, N A)=1}}\left(1-\frac{1}{p^{2}}\right)^{-1}=\alpha^{2} m^{2} \prod_{\substack{p|\alpha m \\ p| N A}}\left(1-\frac{1}{p}\right) \prod_{\substack{p \mid m \\(p, N A)=1}}\left(1+\frac{1}{p}\right)^{-1} .
$$

We observe that

$$
\widetilde{F}_{n}(0)=\frac{1}{2 \pi i} \int_{\left(\frac{3}{4}\right)} \frac{\Gamma(s)}{s}\left(\frac{2 \pi n}{8 Y \sqrt{N}}\right)^{-s} \breve{F}(s) d s
$$


where $\breve{F}(s)=\int_{\Re} F(t) t^{s} d t$. Now substituting these in the above expression for $P(\alpha, \delta)$, and then interchanging the order of summation and integration, we get

$$
P(\alpha, \delta)=Y \frac{c(\alpha, \delta)}{2 \pi i} \int_{\left(\frac{3}{4}\right)} \frac{\Gamma(s)}{s}\left(\frac{8 Y \sqrt{N}}{2 \pi}\right)^{s} \breve{F}(s) L(s+2) d s+O_{\varepsilon}\left(\frac{Y^{1+\varepsilon}}{X \xi \delta}\right),
$$

where

$$
c(\alpha, \delta)=\left(\frac{2 \xi \delta}{\alpha}\right) \frac{1}{2 \xi \delta \alpha} \prod_{(p, 2 N A \xi)=1}\left(1-\frac{1}{p^{2}}\right)
$$

and the $L$-series is given by

$$
L(s)=\sum_{\left(m, 2 \xi \delta_{1}\right)=1} \frac{a_{\alpha m^{2}} \alpha^{2} m^{2}}{\left(\alpha m^{2}\right)^{s}} \prod_{\substack{p|\alpha m \\ p| N A}}\left(1-\frac{1}{p}\right) \prod_{\substack{p \mid m \\(p, N A)=1}}\left(1+\frac{1}{p}\right)^{-1} .
$$

Recall that $\delta_{1}$ is the gcd of $\delta$ and $N A$. Then using the multiplicativity of the Fourier coefficients we obtain the following Euler product expression:

$$
L(s)=\prod_{p \mid \alpha}\left(1-\frac{1}{p}\right) \times\left\{\sum_{\nu \mid \alpha^{\infty}} \frac{a_{\alpha \nu^{2}} \alpha^{2} \nu^{2}}{\left(\alpha \nu^{2}\right)^{s}}\right\} \times \prod_{\substack{p \mid N A \\\left(p, \delta_{1} \alpha\right)=1}} l_{p}(s) \times \prod_{(p, 2 N A \xi)=1} L_{p}(s)^{-1},
$$

where

$$
\begin{aligned}
l_{p}(s) & =\left\{1+\left(1-\frac{1}{p}\right)\left(\frac{a_{p^{2}}}{p^{2(s-1)}}+\frac{a_{p^{4}}}{p^{4(s-1)}}+\cdots\right)\right\}, \\
L_{p}(s)^{-1} & =\left\{1+\left(1+\frac{1}{p}\right)^{-1}\left(\frac{a_{p^{2}}}{p^{2(s-1)}}+\frac{a_{p^{4}}}{p^{4(s-1)}}+\cdots\right)\right\} .
\end{aligned}
$$

The first three terms in the product are absolutely convergent in the domain $\operatorname{Re}(s)>3 / 2$. Also, we note that they are free of the parameter $\xi$. Only the fourth term in the formula above depends on $\xi$. Now

$$
\prod_{(p, 2 N A \xi)=1} L_{p}(s)^{-1}=\left(\prod_{p \mid \xi} L_{p}(s)\right) \mathcal{L}(s) \prod_{(p, 2 N A)=1}\left(L_{p}(s) \mathcal{L}_{p}(s)\right)^{-1},
$$

where

$$
\mathcal{L}_{p}(s)=1+\frac{a_{p^{2}}}{p^{2(s-1)}}+\frac{a_{p^{4}}}{p^{4(s-1)}}+\cdots,
$$

and $\mathcal{L}(s)=\prod_{(p, 2 N A)=1} \mathcal{L}_{p}(s)$, which is holomorphic up to $\operatorname{Re}(s)>7 / 4$. The product $\prod\left(L_{p}(s) \mathcal{L}_{p}(s)\right)^{-1}$ converges absolutely in the region $\operatorname{Re}(s)>3 / 2$. Hence $L(s)$ has analytic continuation up to $\operatorname{Re}(s)>7 / 4$ and can be written as

$$
L(s)=\eta(s) \prod_{p \mid \xi} L_{p}(s),
$$

where $\eta(s)$ depends only on $\alpha$ and $\delta_{1}$, but not on $\xi$. 
Using the analytic properties of $L(s)$, we can evaluate $P(\alpha, \delta)$ by moving the line of integration to $-1 / 4+\varepsilon$ and collecting the residue at the double pole $s=0$. The residue gives the main term $M(\alpha, \delta)$ and the integral at $-1 / 4+\varepsilon$ gives the error $I(\alpha, \delta)$. So we get

$$
P(\alpha, \delta)=M(\alpha, \delta)+I(\alpha, \delta)+O_{\varepsilon}\left(\frac{Y^{1+\varepsilon}}{X \xi \delta}\right) .
$$

Estimation of the error term $I(\alpha, \delta)$ is postponed to the next section. To evaluate the main term we start with the Taylor expansion for the gamma function, and get

$$
\begin{aligned}
L(2+s) & \left(\frac{8 Y t \sqrt{N}}{2 \pi}\right)^{s} \frac{\Gamma(s)}{s} \\
& =\frac{L(2)}{s^{2}}+\frac{1}{s}\left\{L(2) \log \left(\frac{8 Y t \sqrt{N}}{2 \pi}\right)+L^{\prime}(2)-\gamma L(2)\right\}+\cdots,
\end{aligned}
$$

where $\gamma$ is the Euler constant. Hence

$$
M(\alpha, \delta)=Y c(\alpha, \delta)\left\{L(2) \widetilde{F}(0) \log Y+A L(2)+B L^{\prime}(2)\right\},
$$

where $A$ and $B$ are constants which depend only on the elliptic curve $E$ and the smooth function $F$. The constants can be written down explicitly but since that will be of no use to us, we leave them as undetermined. However, it is important to have the formulas for $L(2)$ and $L^{\prime}(2)$ ready at hand:

$$
\begin{aligned}
L(2) & =\eta(2) \prod_{p \mid \xi} L_{p}(2), \\
L^{\prime}(2) & =\eta^{\prime}(2) \prod_{p \mid \xi} L_{p}(2)+L(2) \sum_{p \mid \xi} \frac{L_{p}^{\prime}(2)}{L_{p}(2)} .
\end{aligned}
$$

Also, we note that

$$
\eta(2)=C_{N, A} \prod_{p \mid \alpha} A_{p} \prod_{p \mid \delta_{1}} B_{p}
$$

where

$$
\begin{aligned}
& A_{p}=\left(1-\frac{1}{p}\right)\left(\sum_{j=0}^{\infty} \frac{a_{p^{j+1}}}{p^{2 j}}\right)\left\{1+\left(1-\frac{1}{p}\right)\left(\frac{a_{p^{2}}}{p^{2}}+\frac{a_{p^{4}}}{p^{4}}+\cdots\right)\right\}^{-1}, \\
& B_{p}=\left\{1+\left(1-\frac{1}{p}\right)\left(\frac{a_{p^{2}}}{p^{2}}+\frac{a_{p^{4}}}{p^{4}}+\cdots\right)\right\}^{-1},
\end{aligned}
$$

and $C_{N, A}$ is a nonzero constant which depends only on $N$ and $A$, and is 
given by

$$
\begin{aligned}
C_{N, A}= & \prod_{p \mid N A}\left\{1+\left(1-\frac{1}{p}\right)\left(\frac{a_{p^{2}}}{p^{2}}+\frac{a_{p^{4}}}{p^{4}}+\cdots\right)\right\} \\
& \times \prod_{(p, 2 N A)=1}\left\{1+\left(1+\frac{1}{p}\right)^{-1}\left(\frac{a_{p^{2}}}{p^{2}}+\frac{a_{p^{4}}}{p^{4}}+\cdots\right)\right\} .
\end{aligned}
$$

5. The error terms - I. Now we take up the task of estimating the error terms. In this section we estimate $I(\alpha, \delta)$ and $R(\alpha)$. The estimation of $R(\alpha, \delta)$ is more involved and we prefer to do this in a separate section.

5.1. The term $I(\alpha, \delta)$. Recall that

$$
\begin{aligned}
I(\alpha, \delta)= & \left(\frac{2 \xi \delta}{\alpha}\right) \frac{Y}{2 \xi \delta \alpha} \prod_{(p, 2 N A \xi)=1}\left(1-\frac{1}{p^{2}}\right) \\
& \times \frac{1}{2 \pi i} \int_{(-1 / 4+\varepsilon)} \frac{\Gamma(s)}{s}\left(\frac{8 Y \sqrt{N}}{2 \pi}\right)^{s} \breve{F}(s) L(s+2) d s .
\end{aligned}
$$

Writing $s=-1 / 4+\varepsilon+i t$, and using Phragmén-Lindelöf to the $\operatorname{sym}^{2} L$ function of $E$, we get

$$
\mathcal{L}(2+s) \ll 1+|t|, \quad \prod_{p \mid \xi} L_{p}(2+s) \ll \xi^{\varepsilon},
$$

hence $L(2+s) \ll(1+|t|) \xi^{\varepsilon}$. Using this bound we get

$$
I(\alpha, \delta) \ll \frac{Y}{\xi \delta} Y^{-1 / 4+\varepsilon} \xi^{\varepsilon} \ll \frac{Y^{3 / 4+\varepsilon}}{\xi \delta} .
$$

5.2. The term $R(\alpha)$. Extracting square divisors of $l^{-2} d \xi$, we get

$$
R(\alpha)=\sum_{(b, 2 N A \xi)=1}\left(\sum_{\substack{l \mid b \\ l>X}} \mu(l)\right) \sum_{(d, 2 N A \xi)=1}^{b}\left(\frac{d}{\alpha}\right) \mathcal{A}\left(8 b^{2} d \xi \sqrt{N}, \chi_{8 b^{2} d \xi}\right) F\left(\frac{d b^{2} \xi}{Y}\right) .
$$

Now,

$$
\mathcal{A}\left(z, \chi_{8 b^{2} d \xi}\right)=\frac{1}{2 \pi i} \int_{\left(\frac{3}{4}\right)} \frac{\Gamma(s)}{s} z^{s} L\left(1+s, E \chi_{8 d \xi}\right) \prod_{p \mid b} L_{p}\left(1+s, E \chi_{8 d \xi}\right) d s,
$$

where $L_{p}\left(s, E \chi_{8 d \xi}\right)=\left(1-\chi_{8 d \xi}(p) a_{p} p^{-s}+p^{1-2 s}\right)$. Then we move the line of integration to $\sigma=\varepsilon$; summing over all square-free odd $d \xi$ and applying the Cauchy-Schwarz inequality and the inequality (1) we get

$$
\sum_{(\xi, 2)=1}^{b} \sum_{(d, 2 N A \xi)=1}^{b}\left|\mathcal{A}\left(8 b^{2} d \xi \sqrt{N}, \chi_{8 b^{2} d \xi}\right)\right| F\left(\frac{d b^{2} \xi}{Y}\right) \ll_{\varepsilon} \frac{Y^{1+\varepsilon}}{b^{2}} .
$$


Hence we get the following bound for the average of $R(\alpha)$ :

$$
\sum_{\xi<Y^{\theta}}|R(\alpha)| \ll_{\varepsilon} \sum_{b>0}\left(\sum_{\substack{l \mid b \\ l>X}} 1\right) \frac{Y^{1+\varepsilon}}{b^{2}} \ll_{\varepsilon} \frac{Y^{1+\varepsilon}}{X} .
$$

6. The error terms - II. Now we estimate the error term coming from the nonzero frequencies:

$$
\begin{aligned}
R(\alpha, \delta)= & \sum_{n} \frac{a_{n}}{n} \sum_{\substack{l \leq X \\
(l, 2 N A \xi n)=1}} \mu(l)\left(\frac{2 \xi \delta}{n}\right) \frac{Y}{2 l^{2} \xi \delta \alpha n}\left(\frac{2}{\alpha n}\right) \\
& \times \sum_{k \neq 0}(-1)^{k} G_{k}(\alpha n) \widetilde{F}_{n}\left(\frac{k Y}{2 l^{2} \xi \delta \alpha n}\right) .
\end{aligned}
$$

To analyze this we use the Mellin transform identity

$$
\sum_{n=1}^{\infty} b_{n} g(n)=\frac{1}{2 \pi i} \int_{(c)} \sum_{n=1}^{\infty} \frac{b_{n}}{n^{w}}\left(\int_{0}^{\infty} g(t) t^{w-1} d t\right) d w
$$

where $b_{n} \ll n^{\varepsilon}$ and $g$ is smooth with $g(0)=0$ and rapidly decaying at infinity, and $c>1$. This gives

$$
R(\alpha, \delta)=\left(\frac{2}{\alpha}\right) \sum_{\substack{l \leq X \\(l, 2 N A \xi)=1}} \frac{Y \mu(l)}{2 l^{2} \xi \delta \alpha} \sum_{k \neq 0} \frac{(-1)^{k}}{2 \pi i} \int_{(c)} L_{(k)}(2+w) f\left(\frac{k Y}{2 l^{2} \xi \delta \alpha}, w\right) d w
$$

where

$$
L_{(k)}(w)=\sum_{\substack{n=1 \\(n, 2 l)=1}}^{\infty} \frac{a_{n}}{n^{w}}\left(\frac{\xi \delta}{n}\right) G_{k}(\alpha n), \quad f(\eta, w)=\int_{0}^{\infty} \widetilde{F}_{t}\left(\frac{\eta}{t}\right) t^{w-1} d t .
$$

6.1. The function $L_{(k)}(w)$. To analyze this $L$-series we need to use the following properties of the Gauss sum (details can be found in [15]).

Properties of $G_{k}(n)$

(i) If $n$ is square-free then $G_{k}(n)=\left(\frac{k}{n}\right) \sqrt{n}$.

(ii) (Multiplicativity) Suppose $m$ and $n$ are coprime odd integers. Then $G_{k}(m n)=G_{k}(m) G_{k}(n)$.

(iii) Suppose $p^{\alpha}$ is the largest power of $p$ dividing $k$. (If $k=0$ then set $\alpha=\infty$.) Then, for $\beta \geq 1$, 


$$
G_{k}\left(p^{\beta}\right)= \begin{cases}0 & \text { if } \beta \leq \alpha \text { is odd } \\ \phi\left(p^{\beta}\right) & \text { if } \beta \leq \alpha \text { is even } \\ -p^{\alpha} & \text { if } \beta=\alpha+1 \text { is even } \\ \left(\frac{k p^{-\alpha}}{p}\right) p^{\alpha} \sqrt{p} & \text { if } \beta=\alpha+1 \text { is odd } \\ 0 & \text { if } \beta \geq \alpha+2\end{cases}
$$

Since $(\alpha, l)=1$, from the multiplicativity of the Gauss sum it follows that

$$
L_{(k)}(w)=Q(w) \prod_{(p, 2 l \alpha k N)=1}\left\{1+\frac{a_{p}}{p^{w-1 / 2}}\left(\frac{\xi \delta k}{p}\right)\right\}
$$

where

$$
Q(w)=\left(\sum_{n \mid \alpha^{\infty}} \frac{a_{n}}{n^{w}}\left(\frac{\xi \delta}{n}\right) G_{k}(\alpha n)\right) \prod_{\substack{p \mid k N \\(p, 2 l \alpha)=1}}\left(\sum_{j=0}^{\infty} \frac{a_{p^{j}}}{p^{j w}}\left(\frac{\xi \delta}{p^{j}}\right) G_{k}\left(p^{j}\right)\right) .
$$

Let $\chi_{\xi, \delta, k}$ be the primitive character associated with $p \mapsto\left(\frac{\xi \delta k}{p}\right)$. Then

$$
\prod_{(p, 2 l \alpha k N)=1}\left\{1+\frac{a_{p}}{p^{w-1 / 2}}\left(\frac{\xi \delta k}{p}\right)\right\}=L\left(w-\frac{1}{2}, E, \chi_{\xi, \delta, k}\right) P(w) \Omega(w),
$$

where $P(w)$ is a finite Euler product and

$$
\Omega(w)=\prod_{(p, 2 l \alpha k N)=1}\left\{1-\frac{\chi(p) a_{p}}{p^{w-1 / 2}}+\frac{1}{p^{2 w-2}}\right\}\left\{1+\frac{\chi(p) a_{p}}{p^{w-1 / 2}}\right\} .
$$

From the above we conclude that for $w=-1 / 2+\varepsilon+i t$ we have the bound

$$
L_{(k)}(w+2) \ll\left|L\left(1+\varepsilon+i t, E, \chi_{\xi, \delta, k}\right)\right||\xi l k|^{\varepsilon} .
$$

6.2. The function $f(\eta, w)$. We will not go into details, as most of the calculation is similar to that given in [15]. Interchanging the order of integration we get

$$
f(\eta, w)=|\eta|^{w} \breve{F}(w) \int_{0}^{\infty} V\left(\frac{2 \pi|\eta|}{8 z Y \sqrt{N}}\right)(\cos 2 \pi z+\operatorname{sgn}(\eta) \sin 2 \pi z) \frac{d z}{z^{1+w}} .
$$

Using the contour integral representation of the incomplete gamma function and then interchanging the integrals, we deduce that the above integral is given by

$$
\frac{1}{2 \pi i} \int_{(\widetilde{c})}\left(\frac{8 Y \sqrt{N}}{2 \pi|\eta|}\right)^{s}\left(\cos \frac{\pi}{2}(s-w)+\operatorname{sgn}(\eta) \sin \frac{\pi}{2}(s-w)\right) \frac{\Gamma(s-w)}{(2 \pi)^{s-w}} \Gamma(s) \frac{d s}{s},
$$

where $\widetilde{c}>\max (0, \operatorname{Re}(w))$. From this we get the analytic continuation of $f(\eta, w)$ up to $\operatorname{Re}(w)>-1$. Also, it follows that in the region $1 \geq \operatorname{Re}(w)>-1$ 
we have the following bound:

$$
|f(\eta, w)| \ll(1+|w|)^{-\operatorname{Re}(w)-1 / 2} \exp \left(-\frac{\sqrt{|\eta|}}{10 \sqrt{Y(1+|w|)}}\right)|\eta|^{\operatorname{Re}(w)}|\breve{F}(w)| .
$$

Moving the line of integration to $\widetilde{c}=\varepsilon$ we get the second bound

$$
|f(\eta, w)| \ll_{\varepsilon}(1+|w|)^{\varepsilon}|\eta|^{\operatorname{Re}(w)}|\breve{F}(w)|\left(\frac{8 Y \sqrt{N}}{2 \pi|\eta|}\right)^{\varepsilon} .
$$

To give an upper bound for $R(\alpha, \delta)$, we split the $k$-sum into two parts. For larger $k$ we use the first bound for $f(\eta, w)$ and the trivial bound for the $L$-function. For smaller $k$, our task is more delicate, and we obtain an upper bound on average using the second bound for $f(\eta, w)$.

First, we move the line of integration in the expression for $R(\alpha, \delta)$ to $c=-1 / 2+\varepsilon$ and get

$$
|R(\alpha, \delta)| \ll \frac{Y}{\xi \delta \alpha} \sum_{\substack{l \leq X \\(l, 2 N A \xi \alpha)=1}} \frac{1}{l^{2}} \int_{(c)} \sum_{k \neq 0}\left|L_{(k)}(2+w)\right|\left|f\left(\frac{k Y}{2 l^{2} \xi \delta \alpha}, w\right)\right||d w| .
$$

Taking $\eta=k Y / 2 l^{2} \xi \delta \alpha$ and using the first bound for $f$ and summing over $k$ 's with $|k|>K(w):=1000 l^{2} \xi \delta \alpha(1+|w|)(\log Y)^{4}$, we get

$$
\begin{aligned}
\left|R_{1}(\alpha, \delta)\right| & \ll \frac{Y}{\xi \delta} \sum_{l \leq X} \frac{1}{l^{2}} \int_{(c)} \sum_{|k|>K(w)}\left|L_{(k)}(2+w)\right|\left|f\left(\frac{k Y}{2 l^{2} \xi \delta \alpha}, w\right)\right||d w| \\
& \ll \exp (-\log Y) .
\end{aligned}
$$

For the remaining terms we seek a bound which is true on average over all square-free $\xi<Y^{\theta}$. We write $\delta=\delta_{1} \delta_{2}$ with $\delta_{1} \mid N A$ and $\delta_{2} \mid \xi$, and then define $\delta_{3}$ by $\xi=\delta_{2} \delta_{3}$. Using the second bound for $f$, we get

$$
\begin{aligned}
& \sum_{\xi<Y^{\theta}} \sum_{\delta_{2} \mid \xi}\left|R_{2}(\alpha, \delta)\right| \\
& \ll \sum_{\substack{\delta_{2}, \delta_{3} \\
\delta_{2} \delta_{3}<Y^{\theta}}} \frac{Y^{1 / 2+\varepsilon}}{\delta_{2} \sqrt{\delta_{3}}} \sum_{l \leq X} \frac{1}{l} \int_{(c)} \sum_{k=1}^{K(w)}\left|L_{(k)}(2+w)\right|(1+|w|)|\breve{F}(w)||d w| \\
& \ll Y^{1 / 2+\varepsilon} \sum_{\substack{l \leq X \\
\delta_{2}<Y^{\theta}}} \frac{1}{l \delta_{2}} \int_{(c)}\left(\sum_{\delta_{3}<Y^{\theta} / \delta_{2}} \sum_{k=1}^{K(w)} \frac{\left|L_{(k)}(2+w)\right|}{\sqrt{\delta_{3} k}}\right)(1+|w|)|\breve{F}(w)||d w| .
\end{aligned}
$$

Then observing that $\delta_{3} k<1000 l^{2}(1+|w|)(\log Y)^{4} Y^{2 \theta}$, extracting square-free discriminants from the sum and applying the Cauchy-Schwarz inequality 
and (1), we get

$$
\sum_{\xi<Y^{\theta}} \sum_{\delta_{2} \mid \xi}\left|R_{2}(\alpha, \delta)\right| \ll Y^{1 / 2+\theta+\varepsilon} X^{1+\varepsilon} .
$$

This concludes the proof of our first lemma:

LEMma 5. Let $E$ be an elliptic curve with odd conductor, $N$ say. Let $A$ be a fixed odd integer coprime to $N$, and let $\xi$ be a fixed odd integer coprime to $N A$. For a divisor $\alpha \mid A$, set

$$
S(\alpha)=\sum_{(d, 2 N A \xi)=1} \mu(d)^{2}\left(\frac{d}{\alpha}\right) \mathcal{A}\left(8 d \xi \sqrt{N}, \chi_{8 d \xi}\right) F\left(\frac{d \xi}{Y}\right) .
$$

Then

$$
S(\alpha)=\sum_{\delta_{1} \mid N A} \mu\left(\delta_{1}\right)\left(\frac{\delta_{1}}{\alpha}\right) M\left(\alpha, \delta_{1}\right)+E_{\xi},
$$

where the main term $M\left(\alpha, \delta_{1}\right)$ is given by

$$
M\left(\alpha, \delta_{1}\right)=Y c\left(\alpha, \delta_{1}\right) \frac{\phi(\xi)}{\xi}\left\{L(2) \widetilde{F}(0) \log Y+A L(2)+B L^{\prime}(2)\right\}
$$

with $\widetilde{F}(0), c\left(\alpha, \delta_{1}\right), L(s), A$ and $B$ as given in (2)-(5), and the error term $E_{\xi}$ is bounded on average by

$$
\sum_{\xi<Y^{\theta}}\left|E_{\xi}\right| \ll Y^{3 / 4+\theta / 2+\varepsilon} .
$$

7. A mean value estimate-II. In this section we consider a slightly different sum. Let $\alpha, \xi, A$ and $N$ be as before. Write $N=N_{1} N_{2}^{2}$, where $N_{1}$ is square-free. Then define

$$
T(\alpha)=\sum_{(d, 2 N A \xi)=1} \mu(d)^{2}\left(\frac{d}{\alpha}\right)\left(\frac{d \xi}{N_{1}}\right) \mathcal{A}\left(8 d \xi \sqrt{N}, \chi_{8 d \xi}\right) F\left(\frac{d \xi}{Y}\right) .
$$

This sum can be analyzed in exactly the same way. Only the $L$-series is slightly different and is given by the Euler product

$$
\left\{\sum_{\nu \mid\left(\alpha N_{1}\right)^{\infty}} \frac{a_{\alpha N_{1} \nu^{2}} \alpha^{2} N_{1}^{2} \nu^{2}}{\left(\alpha N_{1} \nu^{2}\right)^{s}}\right\} \prod_{p \mid \alpha N_{1}}\left(1-\frac{1}{p}\right) \prod_{\substack{p \mid N A \\\left(p, \delta_{1} \alpha N_{1}\right)=1}} l_{p}(s) \prod_{(p, 2 N A \xi)=1} L_{p}(s)^{-1} .
$$

We call this function $\widetilde{L}(s)$. This $L$-series has the same analytic properties as $L(s)$, in particular we can write

$$
\widetilde{L}(s)=\widetilde{\eta}(s) \prod_{p \mid \xi} L_{p}(s),
$$

where $\widetilde{\eta}(s)$ depends on $\alpha$ and $\delta_{1}$, but not on $\xi$. 
Lemma 6. We have

$$
T(\alpha)=\left(\frac{\xi}{N_{1}}\right) \sum_{\delta_{1} \mid N A} \mu\left(\delta_{1}\right)\left(\frac{\delta_{1}}{\alpha N_{1}}\right) \widetilde{M}\left(\alpha, \delta_{1}\right)+\widetilde{E}_{\xi}
$$

where $\widetilde{E}_{\xi}$ is bounded on average by $\sum_{\xi \leq Y^{\theta}}\left|\widetilde{E}_{\xi}\right| \ll_{\varepsilon} Y^{3 / 4+\theta / 2+\varepsilon}$, and

$$
\widetilde{M}\left(\alpha, \delta_{1}\right)=Y c\left(\alpha N_{1}, \delta_{1}\right) \frac{\phi(\xi)}{\xi}\left\{\widetilde{L}(2) \widetilde{F}(0) \log Y+A \widetilde{L}(2)+B \widetilde{L}^{\prime}(2)\right\}
$$

where $A$ and $B$ are the same constants as in the previous lemma.

8. The theorem. Now we consider the sequence $\left\{s_{d}\right\}$, where

$$
s_{d}=\mu(d)^{2} \psi_{A}(d)\left(1+\left(\frac{d}{N}\right)\right) L^{\prime}\left(1, E, \chi_{8 d}\right) F\left(\frac{d}{Y}\right) .
$$

If the root number of $E_{8 d}$ is 1 then $s_{d}=0$, otherwise by Gross-Zagier [3] we deduce that $s_{d}$ is nonnegative. Let

$$
S_{\xi}:=\sum_{(d, 2 N A)=1} c_{d \xi}=\sum_{(d, 2 N A \xi)=1} \mu(d)^{2} \psi_{A}(d \xi)\left(1+\left(\frac{d \xi}{N}\right)\right) L^{\prime}\left(1, E, \chi_{8 d \xi}\right) F\left(\frac{d \xi}{Y}\right) .
$$

Our aim is to find the asymptotic behaviour of $S_{\xi}$. Using the approximate functional equation for $L^{\prime}\left(1, E, \chi_{8 d \xi}\right)$ and expanding $\psi_{A}(d \xi)$ we obtain

$$
S_{\xi}=\frac{2}{2^{\nu(A)}} \sum_{\alpha \mid A}\left(\frac{2 \xi}{\alpha}\right)(S(\alpha)+T(\alpha))
$$

where $S(\alpha)$ and $T(\alpha)$ are as in the previous sections. Hence from Lemmas 5 and 6 we get

$$
S_{\xi}=A(\xi) Y \log Y+B(\xi) Y+r_{\xi},
$$

where the error term $r_{\xi}$ is bounded on average by

$$
\sum_{\xi<Y^{\theta}}\left|r_{\xi}\right| \ll_{\varepsilon} Y^{3 / 4+\theta / 2+\varepsilon} .
$$

8.1. Evaluation of $A(\xi)$. From Lemmas 5 and 6 , we get $A(\xi)=A_{1}(\xi) \widetilde{F}(0)$ where

$$
\begin{aligned}
A_{1}(\xi)= & \frac{2}{2^{\nu(A)}} \frac{\phi(\xi)}{\xi}\left\{\sum_{\alpha \mid A} \sum_{\delta_{1} \mid N A}\left(\frac{2 \xi \delta_{1}}{\alpha}\right) \mu\left(\delta_{1}\right) c\left(\alpha, \delta_{1}\right) L(2)\right. \\
& \left.+\sum_{\alpha \mid A} \sum_{\delta_{1} \mid N A}\left(\frac{2 \xi \delta_{1}}{\alpha N_{1}}\right) \mu\left(\delta_{1}\right) c\left(\alpha N_{1}, \delta_{1}\right) \widetilde{L}(2)\right\} .
\end{aligned}
$$


The double sums in the expression for $A(\xi)$ can be evaluated without much trouble using (6), (8) and (9). For example a little work gives

$$
\sum_{\alpha \mid A} \sum_{\substack{\delta_{1} \mid N A \\\left(\delta_{1}, \alpha\right)=1}} \frac{\mu\left(\delta_{1}\right)}{\delta_{1} \alpha} \prod_{p \mid \alpha} A_{p} \prod_{p \mid \delta_{1}} B_{p}=\prod_{p \mid N A} C_{p}^{-1} \prod_{p \mid A}\left(1+\frac{C_{p} A_{p}}{p}\right)
$$

where $C_{p}=\left(1-B_{p} / p\right)^{-1}$. Similarly, we can show that

$$
\sum_{\alpha \mid A} \sum_{\substack{\delta_{1} \mid N A \\\left(\delta_{1}, \alpha N_{1}\right)=1}} \frac{\mu\left(\delta_{1}\right)}{\delta_{1} \alpha N_{1}} \prod_{p \mid \alpha N_{1}} A_{p} \prod_{p \mid \delta_{1}} B_{p}=\frac{1}{N_{1}} \prod_{p \mid N_{1}} A_{p} \prod_{\substack{p \mid N_{2} A \\\left(p, N_{1}\right)=1}} C_{p}^{-1} \prod_{p \mid A}\left(1+\frac{C_{p} A_{p}}{p}\right) .
$$

Writing $D^{*}$ for the sum of the above two terms and setting

$$
C^{*}=\frac{C_{N, A}}{2^{\nu(A)}} \prod_{(p, 2 N A)=1}\left(1-\frac{1}{p^{2}}\right)
$$

we get

$$
A_{1}(\xi)=C^{*} D^{*} \prod_{p \mid \xi} g(p)
$$

where

$$
g(p)=\frac{1}{p}\left(1+\frac{1}{p}+\frac{a_{p^{2}}}{p^{2}}+\frac{a_{p^{4}}}{p^{4}}+\cdots\right)^{-1} .
$$

8.2. Evaluation of $B(\xi)$. From Lemmas 5 and 6 we observe that $B(\xi)=$ $A B_{1}(\xi)+B B_{2}(\xi)$, where $B_{1}(\xi)=A_{1}(\xi)$. So it remains to evaluate $B_{2}(\xi)$, which is given by

$$
\begin{aligned}
\frac{2}{2^{\nu(A)}} \frac{\phi(\xi)}{\xi}\left\{\sum_{\alpha \mid A} \sum_{\delta_{1} \mid N A}\left(\frac{2 \xi \delta_{1}}{\alpha}\right) \mu\left(\delta_{1}\right) c\left(\alpha, \delta_{1}\right) L^{\prime}(2)\right. \\
\\
\left.\quad+\sum_{\alpha \mid A} \sum_{\delta_{1} \mid N A}\left(\frac{2 \xi \delta_{1}}{\alpha N_{1}}\right) \mu\left(\delta_{1}\right) c\left(\alpha N_{1}, \delta_{1}\right) \widetilde{L}^{\prime}(2)\right\} .
\end{aligned}
$$

Again, the contribution of the part coming from $\eta^{\prime}(2)$ and $\widetilde{\eta}^{\prime}(2)$ (see (7), (9)) can be computed as in the previous subsection. The remaining part is given by

$$
\begin{aligned}
\frac{2}{2^{\nu(A)}} \frac{\phi(\xi)}{\xi}\left\{\sum_{\alpha \mid A}\right. & \sum_{\delta_{1} \mid N A}\left(\frac{2 \xi \delta_{1}}{\alpha}\right) \mu\left(\delta_{1}\right) c\left(\alpha, \delta_{1}\right) L(2) \\
& \left.+\sum_{\alpha \mid A} \sum_{\delta_{1} \mid N A}\left(\frac{2 \xi \delta_{1}}{\alpha N_{1}}\right) \mu\left(\delta_{1}\right) c\left(\alpha N_{1}, \delta_{1}\right) \widetilde{L}(2)\right\} \sum_{p \mid \xi} \frac{L_{p}^{\prime}(2)}{L_{p}(2)},
\end{aligned}
$$


which is equal to

$$
A_{1}(\xi) \sum_{p \mid \xi} \frac{L_{p}^{\prime}(2)}{L_{p}(2)}
$$

Finally, we observe that

$$
\frac{L_{p}^{\prime}(2)}{L_{p}(2)}=\left(1+\frac{1}{p}+\frac{a_{p^{2}}}{p^{2}}+\frac{a_{p^{4}}}{p^{4}}+\cdots\right)^{-1}\left(\frac{2 a_{p^{2}}}{p^{2}}+\frac{4 a_{p^{4}}}{p^{4}}+\cdots\right)(\log p) .
$$

Acknowledgements. The results presented in this article are based on chapters three and four of my thesis [10]. It is a pleasure to thank my advisor Prof. Andrew Wiles for many helpful discussions and his interest in this work. I also thank Prof. Peter Sarnak for many insightful comments and for advising me to study sieve theory. Finally, I thank Prof. Henryk Iwaniec for his guidance.

\section{References}

[1] D. Bump, S. Friedberg and J. Hoffstein, Eisenstein series on the metaplectic group and nonvanishing theorems for automorphic L-functions and their derivatives, Ann. of Math. (2) 131 (1990), 53-127.

[2] G. Greaves, Sieves in Number Theory, Ergeb. Math. Grenzgeb. (3) 43, Springer, Berlin, 2001.

[3] B. H. Gross and D. B. Zagier, Heegner points and derivatives of L-series, Invent. Math. 84 (1986), 225-320.

[4] H. Halberstam and H.-E. Richert, Sieve Methods, London Math. Soc. Monogr. 4, Academic Press, 1974.

[5] D. R. Heath-Brown, A mean value estimate for real character sums, Acta Arith. 72 (1995), 235-275.

[6] J. Hoffstein and W. Luo, Nonvanishing of L-series and the combinatorial sieve, Math. Res. Lett. 4 (1997), 435-444.

[7] H. Iwaniec, On the order of vanishing of modular L-functions at the critical point, Sém. Théorie Nombres Bordeaux 2 (1990), 365-376.

[8] —, Rutgers notes on sieves, 1996.

[9] K. James, L-series with nonzero central critical value, J. Amer. Math. Soc. 11 (1998), 635-641.

[10] R. Munshi, The arithmetic of elliptic fibrations, doctoral dissert., Princeton Univ., 2006.

[11] M. R. Murty and V. K. Murty, Mean values of derivatives of modular L-series, Ann. of Math. (2) 133 (1991), 447-475.

[12] K. Ono, Nonvanishing of quadratic twists of modular L-functions and applications to elliptic curves, J. Reine Angew. Math. 533 (2001), 81-97.

[13] K. Ono and C. Skinner, Non-vanishing of quadratic twists of modular L-functions, Invent. Math. 134 (1998), 651-660.

[14] A. Perelli and J. Pomykała, Averages of twisted elliptic L-functions, Acta Arith. 80 (1997), 149-163. 
[15] K. Soundararajan, Nonvanishing of quadratic Dirichlet $L$-functions at $s=1 / 2$, Ann. of Math. (2) 152 (2000), 447-488.

[16] V. Vatsal, Rank-one twists of a certain elliptic curve, Math. Ann. 311 (1998), 791794.

[17] A. Wiles, Modular elliptic curves and Fermat's last theorem, Ann. of Math. (2) 141 (1995), 443-551.

Department of Mathematics

Rutgers University

110 Frelinghuyen Road

Piscataway, NJ 08854, U.S.A.

E-mail: rmunshi@math.rutgers.edu

Received on 16.4.2007

and in revised form on 20.1.2009 